This is an author produced version of a paper published in Chemosphere. This paper has been peer-reviewed but does not include the final publisher proof-corrections or journal pagination.

Citation for the published paper:

Rylander, Lars and Nilsson-Ehle, Peter and Hagmar, Lars

"A simplified precise method for adjusting serum levels of persistent organohalogen pollutants to total serum lipids."

Chemosphere. 2006 Jan;62(3):333-6

http://dx.doi.org/10.1016/j.chemosphere.2005.04.107

Access to the published version may require journal subscription.

Published with permission from: Elsevier 


\section{A simplified precise method for adjusting serum levels of persistent organohalogen pollutants to total serum lipids}

Lars Rylander ${ }^{1}$, Peter Nilsson-Ehle ${ }^{2}$, Lars Hagmar ${ }^{1}$

1) Department of Occupational and Environmental Medicine, Institute of Laboratory Medicine, Lund University Hospital, Lund, Sweden

2) Department of Clinical Chemistry, Institute of Laboratory Medicine, Lund University Hospital, Lund, Sweden

Correspondence and reprint requests to:

Associate professor Lars Rylander

Department of Occupational and Environmental Medicine,

Institute of Laboratory Medicine, University Hospital, SE-221 85 Lund, Sweden

Tel: +46 4622233 17; Fax: +46461736 69

Email: lars.rylander@ymed.lu.se 


\section{Abstract}

In order to obtain biomarker values that reflect body burden of persistent organohalogen pollutants (POP), concentrations of lipophilic POP in serum or plasma are generally expressed on a lipid weight basis, and not on a fresh weight basis. There are two different approaches to determine the lipid content in serum and plasma. The gravimetric determination is more expensive, and longer experience is needed as compared with the preferable enzymatic determination. Clinical chemistry laboratories at most hospitals perform enzymatic determinations of cholesterol and triglycerides on a routine basis, whereas analysis of phospholipids is not part of these analyses. In the present study, therefore, we evaluated the association between the sum of measured triglyceride, cholesterol, and phospholipid levels in serum among 617 individuals with the sum of only triglyceride and cholesterol levels. There was a very strong linear association between the sums of serum concentrations of triglycerides and cholesterols and the total lipid concentrations. As much as $97.2 \%$ of the variation in total lipid concentrations was explained by the sum of the triglyceride and cholesterol concentrations, with the following regression: Total lipid $=0.9+1.3 *($ Cholesterol + Triglycerides). Almost identical relationships were recorded in subgroups with men, women and subjects of different age. Thus, total lipid concentrations in blood serum can be accurately estimated by analyzing only cholesterol and triglycerides in the matrix.

Keywords: triglycerides, cholesterol, phospholipids, PCB, dioxin, regression 


\section{Background}

Blood serum and plasma are the most frequently used matrices to quantitate biomarkers of human exposure to highly lipophilic persistent organohalogen pollutants (POP), such as polychlorinated dibenzo-p-dioxins (PCDD), polychlorinated dibenzofurans (PCDF), polychlorinated biphenyls (PCB) and polybrominated diphenylethers. The main body burden of these compounds is found in adipose tissue (Brouwer et al., 1995). The distribution of POP among tissues is in dynamic equilibrium, which means that a change in the lipid content of one tissue would alter its content of POP. As a consequence, postprandial increase in serum lipids will proportionally affect serum levels of POP. This was elegantly shown by Phillips et al (1989), who found that a $20 \%$ increase in total serum lipids led to similar increases in serum concentrations of several different POPs. In order to obtain biomarker values that reflects body burden, it is therefore usual that the concentrations of lipophilic POP in serum or plasma are generally related to its lipid content, i.e. expressed as ng/g lipid rather than $\mu \mathrm{g} / \mathrm{L}$ (Brown and Lawton, 1984).

Two different approaches to determine the lipid content in serum and plasma are commonly used. One alternative utilizes extraction with solvents followed by solvent evaporation and gravimetric determination of the residual in order to obtain the total lipid weight. The other approach is to determine the concentrations of individual lipid classes such as cholesterol, triglycerides and phospholipids by chemical (most frequently enzymatic) methods. These amounts are then summarized using estimations of the average molecular weight of the lipid classes (Cheek and Wease, 1969; Philipps et al., 1989; Akins et al., 1989; Grimvall et al., 1997). Long experience with gravimetric determinations is needed in order to obtain an analytical quality comparable with that for enzymatic determinations (Grimvall et al., 1997). Furthermore, enzymatic determinations are cheaper and less laborious than gravimetric ones. Thus, the enzymatic summation method is to be preferred.

Enzymatic determinations of cholesterol and triglycerides are made on a routine basis for clinical purposes at clinical chemistry laboratories at most hospitals. On the other hand, analysis of phospholipids in serum or plasma is not a part of routine clinical chemistry, and these analyses are therefore less accessible. If a good estimate of total lipids could be obtained based on only determinations of triglycerides and cholesterol, this would be of a great practical value for laboratories performing chemical POP analyses. Phillips et al have presented a simplified formula for calculation of total lipids based on measured cholesterol and triglyceride levels and on an estimate for phospholipids based on the triglyceride concentration (1989). The comparison study was based on 81 subjects. Unfortunately no direct comparison was made 
between the total lipid concentrations based on all three measured substances and the estimates based on the sum of measured triglyceride and cholesterol levels and the estimated phospholipid concentration.

We have access to large materials of enzymatic determinations of cholesterol, triglycerides and phosholipids in human serum, which can be used to evaluate in more detail if an accurate estimate of total lipids can be obtained based on only cholesterol and triglyceride levels. The aim of the present study was therefore to evaluate the associations between the sum of measured triglyceride, cholesterol, and phospholipid levels in serum with the sum of only triglyceride and cholesterol levels, and to define a transformation coefficient from the regression equation, to be used for estimating total lipids in serum.

\section{Material and methods}

\subsection{Available serum samples}

The samples were obtained from four different populations in Sweden and includes almost all individuals who participated in the previous studies (table 1) (Sjödin et al., 1999; Richthoff et al., 2003; Axmon et al., 2004; Rignell-Hydbom et al., 2004). The populations have been described in detail in previous studies. The reason for determining the lipid concentrations in these individuals was to estimate POP concentrations on a lipid weight basis. In total, the study base includes 617 individuals. The women's median age was 44 years (range 29-55). The age distribution among the men was bimodal. Samples were obtained from a large group of men, 18-21 years of age, at a pre-conscript medical examination. The other men ranged in age between 24 and 66 years (median 47). There was no requirement of a fasting period before blood sampling.

\subsection{Enzymatic lipid determination in serum}

Concentrations of triglycerides, cholesterol and phospholipids were determined by enzymatic methods using reagents from Boehringer-Mannheim (triglycerides and cholesterol; Mannheim, Germany) and Waco Chemicals (phospholipids; Neuss, Germany). The total lipid concentration was calculated by summation of the amounts of triglycerides, cholesterol and phospholipids. In these calculations, the average molecular weights of triglycerides and phospholipids were assumed to be 807 and 714. For cholesterol we used an average molecular weight of 571, assuming that the proportion of free and esterified cholesterol serum was 1:2. The long-term, inter-assay coefficient of variation of cholesterol and triglyceride determinations was 2-4 \% and that of phospholipid measurements 5-7 \%. 


\subsection{Statistical methods}

Linear regression models were applied to estimate the association between the sum of serum concentrations of triglycerides and cholesterols and the total lipid concentrations (i.e. the sum of serum concentrations of triglycerides, cholesterol and phospholipids). The effect of age was evaluated. In addition, separate analyses were performed for men and women. Model assumptions were checked by means of residual analysis.

\section{Results}

There was a strong linear association between the sums of serum concentrations of triglycerides and cholesterols and the total lipid concentrations (figure 1). In the present data, as much as $97.2 \%$ of the variation in total lipid concentration was explained by the variation of the sum of the triglyceride and cholesterol concentrations (table 2). The slope was similar for the women (slope $[\beta]=1.31,95 \%$ confidence interval $[\mathrm{CI}] 1.27-1.35)$ and for the men $(\beta=1.28,95 \% \mathrm{CI}$ 1.26-1.29). In addition, the explained variance was also very similar (95.9\% and $98.4 \%$, respectively). Although age was significantly $(\mathrm{p}<0.001)$ associated with the total lipid concentrations it was of minor importance for the estimations. Including age in the regression models did only change the slopes marginally (women age adjusted $\beta=1.30$, 95\% CI 1.25-1.34; men age adjusted $\beta=1.25$, 95\% CI 1.22-1.27) and did not really affect the explained variances (women 96.0\%; men 98.5\%).

\section{Discussion}

The results of the present study show that total lipid concentrations in blood serum can be precisely estimated by analyzing only cholesterol and triglycerides in the matrix. The results can be converted to total lipids using the regression coefficients described. The association was linear over the whole lipid concentration range and essential identical for men and women and for different age groups.

We have earlier shown (Grimvall et al., 1997) that enzymatic measurements of triglycerides, cholesterol and phospholipids accurately reflect gravimetrically determined lipid concentrations in plasma. The present study demonstrates that similar information can be obtained by measurements of triglycerides and cholesterol only. It is evident that such a simplification also leads to an improvement of the precision and reproducibility of the lipid estimation. The total, long-term variation of the triglyceride + cholesterol determinations (square root of the summed squares for the CVs for individual compounds) can be estimated at about 4-5 \% (CV), whereas the variation of triglyceride + cholesterol + phospolipid measurements can be calculated at 7 - 
$8 \%$. This is minor compared to the biological variation of plasma lipid concentrations (if relevant considering the dynamic equilibrium of POPs) and, at any rate, considerably lower than the imprecision of POP measurements.

We think that it is justifiable to use the overall regression equation (total lipid $=0.9+1.3$ * [cholesterol + trigycerides]) for calculating total lipids, because the error introduced by not adjusting for age or gender, is so very minor compared to the measurement error of the POP compounds. However, for those who prefer more specific equations, sufficient information is provided (table 2).

The major drawback with enzymatic determinations of total lipids has been the difficulty to get access to a routine method for phospholipids. Our results show that this drawback is now overcome since accurate estimations of total lipids can be obtained based on the routine methods for cholesterol and triglycerides, which are available at every hospital based clinical chemistry laboratory. It is reasonable to assume that the overall accuracy for lipid weight based POP determinations would be enhanced if the analytical chemistry laboratories performing POP analyses in the future will buy the relatively cheap cholesterol and triglyceride analyses from clinical chemistry laboratories, which have continuous quality control programs running to ensure the long-term performance of these analyses.

\section{Acknowledgements}

Financial support was given by the European Commission RD Life Science Program (QLK4CT-2000-0261 and QLK4-CT-2001-00202), the Swedish Research Council for Medicine, the Swedish Research Council for Environment, Agricultural Sciences and Spatial Planning, the Medical Faculty, Lund University, and Region Skåne funds. 


\section{References}

Akins, J.R., Waldrep, K., Bernert, J.T. Jr., 1989. The estimation of total serum lipids by a completely enzymatic 'summation' method. Clin. Chim. Acta 184, 219-226

Axmon, A., Rylander, L., Strömberg, U., Jönsson, B., Nilsson-Ehle, P., Hagmar, L., 2004. Polychlorinated biphenyls in serum and time to pregnancy. Environ. Res. 96, 186-195.

Brouwer, A., Ahlborg, U.G., Van den Berg, M., Birnbaum, L., Boersma, E.R., Bosveld, B., Denison, M.S., Gray, L.E., Hagmar, L., Holene, L., Huisman, M., Jacobson, S.W., Jacobson, J.L., Koopman-Esseboom, C., Koppe, J.G., Kulig, B.M., Morse, D.C., Muckle, G., Peterson, R.E., Sauer, P.J., Seegal, R.F., Smits-Van Prooije, A.E., Touwen, B.C., Weisglas-Kuperus, N., Winneke, G., 1995. Functional aspects of developmental toxicity of polyhalogenated aromatic hydrocarbons in experimental animals and human infants. Eur. J. Pharmacol. Environ. Toxicol. Pharmacol. Sec., 1-40.

Brown, JF., Lawton, RW., 1984. Polychlorinated biphenyls (PCB) partitioning between adipose tissue and serum. Bull. Environ. Contam. Toxicol., 33, 277-280.

Cheek, C., Wease, D., 1969. A summation technique for serum lipids. Comparison of Methods. Clin. Chem., 15, 102-107.

Grimvall, E., Rylander, L., Nilsson-Ehle, P., Nilsson, U., Strömberg, U., Hagmar, L., Östman C., 1997. Monitoring of polychlorinated biphenyls in human blood plasma: Methodological developments and influence of age, lactation, and fish consumption. Arch. Environ. Contam. Toxicol. 32, 329-336.

Phillips, D.L., Pirkle, J.L., Burse, V.W., Bernert, J.T., Henderson, L.O., Needham, L.L., 1989. Chlorinated hydrocarbon levels in human serum: effects of fasting and feeding. Arch. Environ. Contam. Toxicol. 18, 495-500.

Richthoff, J., Rylander, L., Jönssson, B.A.G., Åkesson, H., Hagmar, L., Nilsson-Ehle, P., Stridsberg M., Giwercman A., 2003. Serum levels of 2,2',4,4’,5,5’hexachlorobiphenyl (CB-153) in relation to markers of reproductive function in young males from general Swedish population. Environ. Health. Perspect. 111, 409-413.

Rignell-Hydbom, A., Rylander, L., Giwercman, A., Jönsson, B.A.G., Nilsson-Ehle, P., Hagmar, L., 2004. Exposure to CB-153 and p,p’-DDE and male reproductive function. Hum. Reprod. 19, 2066-2075.

Sjödin, A., Hagmar, L., Klasson-Wehler, E., Kronholm-Diab, K., Jakobsson, E., Bergman, Å., 1999. Flame retardant exposure: polybrominated diphenyl ethers in blood from Swedish workers. Environ. Health. Perspect. 107, 643-648. 
Table 1. Four different Swedish study populations that have donated blood samples for determination of POP and total lipids using enzymatic methods.

\begin{tabular}{|c|c|c|c|c|}
\hline \multirow[b]{3}{*}{ Study populations } & \multicolumn{2}{|r|}{ Men } & \multicolumn{2}{|c|}{ Women } \\
\hline & \multicolumn{2}{|r|}{ Age } & \multicolumn{2}{|r|}{ Age } \\
\hline & $\mathrm{N}$ & median (range) & $\mathrm{N}$ & median (range) \\
\hline Fishermen $^{\mathrm{a}}$ & 95 & $49 \quad(31,66)$ & - & - \\
\hline Fishermen’s sisters $^{\mathrm{b}}$ & - & - & 180 & $44 \quad(29,55)$ \\
\hline Electronics dismantlers ${ }^{c}$ & 38 & $42(24,60)$ & - & - \\
\hline Conscripts $^{\mathrm{d}}$ & 304 & $18(18,21)$ & - & - \\
\hline Total & 437 & $18(18,66)$ & 180 & $44 \quad(29,55)$ \\
\hline
\end{tabular}

${ }^{a}$ Rignell- Hydbom et al., 2004.

${ }^{\mathrm{b}}$ Axmon et al., 2004.

${ }^{c}$ Sjödin et al., 1999.

${ }^{\mathrm{d}}$ Richthoff et al., 2003.

Table 2 The associations between the sum of serum concentrations of triglycerides (Tg, g/L) and cholesterol (Chol, g/L) and the total lipid concentrations (i.e. the sum of serum concentrations of triglycerides, cholesterol and phospholipids, g/L) in samples from 617 Swedish subjects, obtained from linear regression model. The effect of age was also evaluated. In addition, explained variance $\left(\mathrm{R}_{2}\right)$ is presented.

\begin{tabular}{|c|c|}
\hline Regression models & $\mathrm{R}_{2}$ \\
\hline \multicolumn{2}{|l|}{ Women } \\
\hline Total lipid $=1.13+1.31 *($ Chol + Tg $)$ & 0.959 \\
\hline Total lipid $=0.84+1.30 *($ Chol + Tg $)+0.008 *$ Age & 0.960 \\
\hline \multicolumn{2}{|l|}{ Men } \\
\hline Total lipid $=0.96+1.28 *(\mathrm{Chol}+\mathrm{Tg})$ & 0.984 \\
\hline Total lipid $=0.97+1.25 *($ Chol + Tg $)+0.004 *$ Age & 0.985 \\
\hline \multicolumn{2}{|l|}{ All } \\
\hline Total lipid $=0.92+1.31 *($ Chol $+\mathrm{Tg})$ & 0.972 \\
\hline Total lipid $=0.90+1.24 *($ Chol + Tg $)+0.009 *$ Age & 0.975 \\
\hline
\end{tabular}


Figure The sum of serum concentrations of triglycerides and cholesterols and the total lipid concentrations (i.e. the sum of serum concentrations of triglycerides, cholesterol and phospholipids) in samples from 617 Swedish subjects. In addition, the regression line is shown.

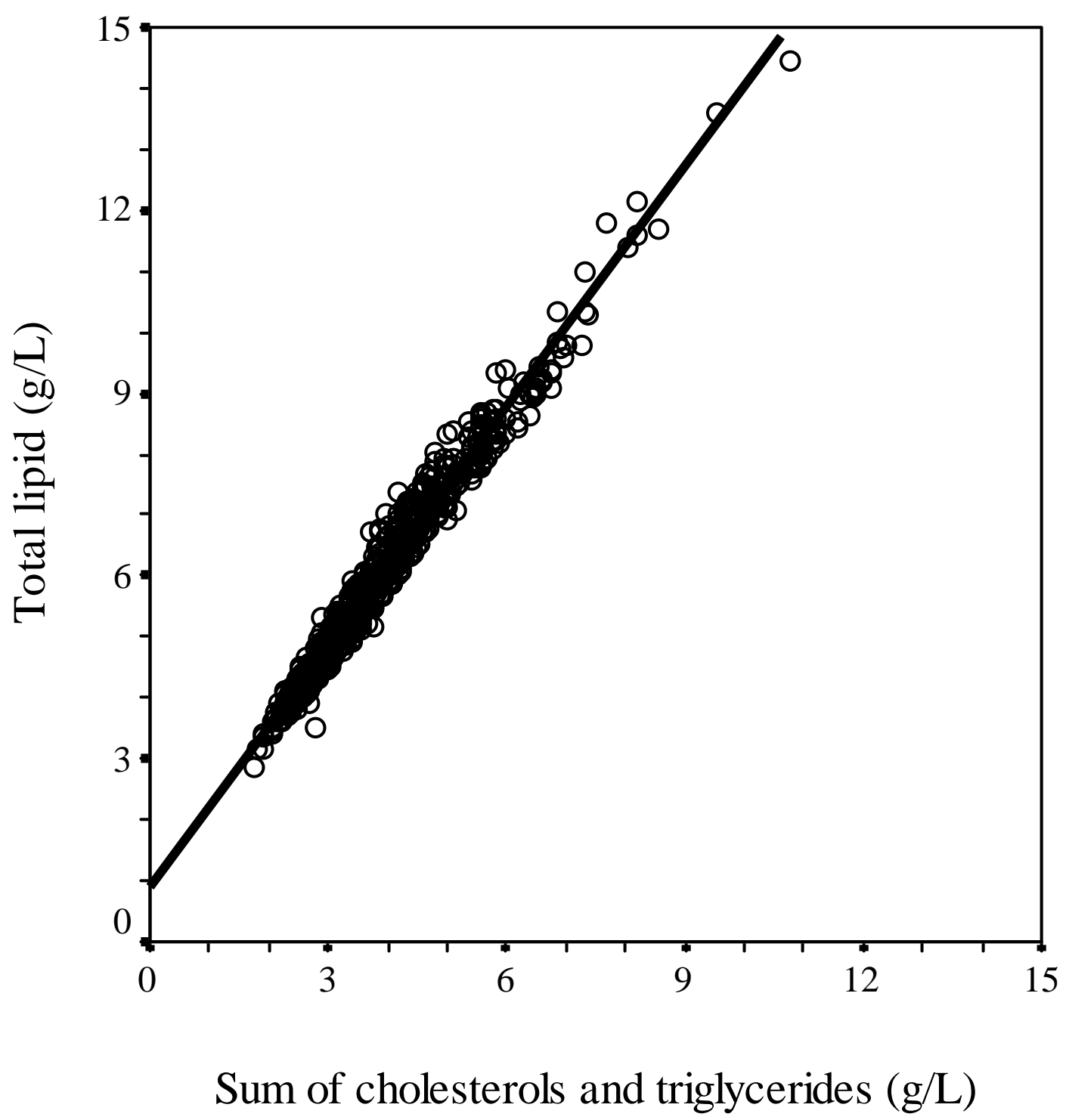

